\title{
ON THE GOORDINATIZATION THEOREM OF J. VON NEUMANN
}

\author{
K. D. FRYER AND ISRAEL HALPERIN
}

1. Introduction. A classical theorem of $n$-dimensional projective geometry asserts: if Desargues' theorem holds (in particular, if $n \geqslant 3$ ) then the points of the geometry can be assigned homogeneous coordinates $\left(\alpha^{1}, \ldots, \alpha^{n+1}\right)$ with $\alpha^{i}$ not all zero in a suitable division ring $\Re(2$, p. $104 ; 3$, Theorems 10 , 11, ex. 19, p. 204). A deep generalization by John von Neumann (5, Theorem 14.1, p. 141) proved such a theorem for every complemented modular lattice possessing a homogeneous basis of $n+1$ elements, $n \geqslant 3$. (The von Neumann theorem provides coordinates for all the elements in the lattice, so that in the special case of the projective geometry, coordinates are provided not merely for points, but for all points, lines, planes, etc. In the general complemented modular lattice there need not be "points".) For the general complemented modular lattice the ring $\Re$ may be an arbitrary regular ring with unit. ${ }^{1}$

A precise statement of the von Neumann theorem is as follows:

Let $L$ be a complemented modular lattice possessing a homogeneous basis ${ }^{2}$ $a_{1}, \ldots, a_{n}, n \geqslant 4$, and let $L_{12}$ be the set of all inverses of $a_{2}$ with respect to $a_{1}+a_{2}$. Then (i) addition and multiplication can be defined for the elements of $L_{12}$ in such a way that $L_{12}$ becomes a regular ring $\Re$ and the sub-lattice $L\left(a_{1}\right)$, consisting of all $x \leqslant a_{1}$, is isomorphic to the lattice of all left principal ideals of $\Re$, and (ii) $L$ is lattice-isomorphic to (coordinatized by) the lattice of all left principal ideals of $\Re_{n}$.

Here $\Re_{n}$ consists of all $n \times n$ matrices with elements in $\Re$ and, as shown by von Neumann, is regular along with $\Re$. It was shown by von Neumann that (ii) above is equivalent to the more classical form of coordinatization, (ii) ${ }^{\prime} L$ is lattice-isomorphic to the lattice of $\Re$-left modules of finite span in the space $V$ of vectors $v=\left(\alpha^{1}, \ldots, \alpha^{n}\right)$ with all $\alpha^{i}$ in $\Re$.

The definitions of addition and multiplication as originally given by von

Received August 11, 1954.

${ }^{1}$ Von Neumann (5, Chapter 2) calls a ring regular if for each $x$ there is a $y$ satisfying $x y x=x$.

${ }^{2}$ Lattice elements $a_{1}, \ldots, a_{m}$ are called $i$ dependent if $\left(a_{1}+\ldots+a_{i-1}\right) a_{i}=0$ for $i=2, \ldots$, $m$. A relative complement or inverse of $x$ in $z$ is any element $[z-x]$ for which $x \oplus[z-x]=z$ $(\oplus$ is used to indicate that the addends are independent). Lattice elements $x$ and $y$ are called perspective, written $x \sim y$, if there exists any $z$, called an axis of perspectivity, such that $x \oplus z=y \oplus z$. Independent elements $a_{1}, \ldots, a_{n}$ with $a_{1}+\ldots+a_{n}=1$ are said to form a basis for $L$; if also $a_{i} \sim a_{j}$ for each $i, j$, the basis is called homogeneous; a homogeneous basis $a_{1}, \ldots, a_{n}$ together with axes of perspectivity $c_{i j}$ with the properties:

$$
c_{i j} \oplus a_{i}=c_{i j} \oplus a_{j} ; c_{i j}=c_{j i} ; c_{i i}=0 ;\left(c_{i j}+c_{j k}\right)\left(a_{i}+a_{k}\right)=c_{i k} ;
$$

is called a normalized frame for $L$. The collection of all inverses of $a_{j}$ in $a_{i}+a_{j}$ will be denoted by $L_{i j}$. $(1$, Sections $2.3,2.4,2.5,4.1 ; 4$, Chapter $2 ; \mathbf{5}$, Definitions $3.1,3.3,5.2$. 
Neumann for (i) above, were motivated in part by the classical constructions in projective geometry, but the verification that $\Re$ becomes a regular ring was based on a rather involved analysis. A simplified discussion of this part of von Neumann's theorem was given in (1).

Von Neumann proved (ii) by showing that for any $m=1, \ldots, n-1$, a given isomorphism between the lattice spanned by $a_{1}, \ldots, a_{m}$ and the lattice of left principal ideals of $\Re_{m}$ can be extended from $m$ to $m+1$, with a special discussion for the extension from $m=n-1$ to $m=n$.

The object of this paper is to give a direct proof of (ii)' (equivalent, as pointed out above, to (ii)) which does not use matrix rings or extension theorems.

As in $(5$, p. 32 and p. $64 ; \mathbf{1}, \$ \$ 4.1$ and 4.3$)$ we shall assume a fixed normalized frame for $L(n \geqslant 4)$ and $\Re$ will consist of all $L$-numbers $\alpha=\left\langle\alpha_{i j} ; i, j=1, \ldots, n\right.$, $i \neq j\rangle$ for which each $\alpha_{i j}$ is in the corresponding $L_{i j}$ and the identities hold: $\left(\alpha_{i j}+c_{j k}\right)\left(a_{i}+a_{k}\right)=\alpha_{i k}$ and $\left(\alpha_{i j}+c_{i k}\right)\left(a_{j}+a_{k}\right)=\alpha_{k j}$. $V$ will denote the set of all vectors of length $n$ with coordinates in $\Re$; a subscript $l$ will denote either left ideal in $\Re$ or $\Re$-left module in $V$.

We shall give a rule which assigns to each element $x$ in $L$ a family of left modules in $V$. Then we shall show that all left modules assigned by this rule to $x$ actually coincide and we shall verify that the rule sets up a $(1,1)$ order preserving correspondence (i.e., a lattice isomorphism) between $L$ and the set of all left modules of finite span. This will establish the von Neumann coordinatization theorem.

2. Notation. We shall adopt the notation:

$$
\begin{array}{lr}
A^{0}=0 ; A^{i}=a_{1}+\ldots+a_{i}, & i=1, \ldots, n ; \\
A^{i}{ }_{j}=a_{1}+\ldots+a_{j-1}+a_{j+1}+\ldots+a_{i}, & 0 \leqslant j \leqslant i \leqslant n .
\end{array}
$$

A system of lattice elements $\langle b\rangle=\left\langle b_{i j} ; i, j=1, \ldots, n, i \neq j\right\rangle$, satisfying $b_{i j} \leqslant a_{i}+a_{j}$, will be called a fraternal system if the identities hold: $\left(b_{i j}+c_{j k}\right)\left(a_{i}+a_{k}\right)=b_{i k} ;\left(b_{i j}+c_{i k}\right)\left(a_{k}+a_{j}\right)=b_{k j}$; if any, and hence each, of the $b_{i j}$ is in the corresponding $L_{i j}$, the fraternal system coincides with an $L$ number. Von Neumann's work shows that for each $x \leqslant a_{i}+a_{j}$ there is one and only one fraternal system $\langle b\rangle$ with $b_{i j}=x(5$, Lemma $6.1 ; 1, \S 4.3)$.

For future reference we list the following formulae:

$$
\begin{aligned}
& (\alpha+\beta)_{i j}=\left[\alpha_{k j}+\left(\beta_{i j}+a_{k}\right)\left(c_{i k}+a_{j}\right)\right]\left(a_{i}+a_{j}\right), \\
& (\alpha+\beta)_{i j}=\left[\left(\alpha_{i k}+a_{j}\right)\left(\beta_{i j}+a_{k}\right)+c_{j k}\right]\left(a_{i}+a_{j}\right), \\
& (\alpha-\beta)_{k j}=\left[\alpha_{i j}+\left(a_{k}+\beta_{i j}\right)\left(a_{j}+c_{i k}\right)\right]\left(a_{k}+a_{j}\right), \\
& (\alpha \beta)_{i j}=\left(\alpha_{i k}+\beta_{k j}\right)\left(a_{i}+a_{j}\right) .
\end{aligned}
$$

The formulae (2.1), (2.2), (2.3), and (2.4) may be obtained from (3, Chap. 7); they are given explicitly in (1), numbered as (4.9.1), (4.9.2), (4.9.3), and (4.10.1) respectively. 
3. Reach and nullity. We associate with each $\alpha$ in $\Re$ a fraternal system which we shall call the nullity of $\alpha$, defined as

$$
\langle b\rangle \text { with } b_{i j}=\alpha_{i j} a_{i} \text { for all } i, j \text {. }
$$

It follows from the fraternal character of the $\alpha_{i j}$ that the $\alpha_{i j} a_{i}$ do form a fraternal system; moreover $b_{i j} \leqslant a_{i}$ for all $i, j$, and hence is independent of $j$. We shall write $\alpha^{0}{ }_{i}$ for this $b_{i j}$.

For each $\alpha$ in $\Re$, the reach of $\alpha$ is defined as in $(1, \S 4.13)$ to be the fraternal system $\langle b\rangle$ with $b_{i j}=\left(\alpha_{i j}+a_{i}\right) a_{j}$, written as $\alpha^{r}{ }_{j}$, identical with the $(\alpha)_{j}$ of von Neumann (5, Definition 9.1). We shall use the following:

(3.1) For every $b \leqslant a_{j}$ and $d=\left[a_{j}-b\right]$ there is an idempotent $e$ in $\Re$ with $e^{r}{ }_{j}=b$ and $^{2}(1-e)^{r}{ }_{j}=d$.

(3.2) For $\alpha, \beta$ in $\Re$ there is a $\gamma$ satisfying $\gamma \alpha=\beta$ if and only if $\alpha^{r}{ }_{j} \geqslant \beta^{r}{ }_{j}$.

For proofs of (3.1) and (3.2) see (5, Theorem 9.3, Lemma 9.1; 1, §4.13).

We shall now prove the following relations:

(3.3) $\alpha \beta=0$ if and only if $\alpha^{r}{ }_{j} \leqslant \beta^{0}{ }_{j}$;

(3.4) $\quad e^{0}{ }_{j}=(1-e)^{r}$ for every idempotent $e$;

(3.5) $\quad e^{0}{ }_{j} \oplus(1-\mathrm{e})^{0}{ }_{j}=e^{0}{ }_{j} \oplus e^{r}{ }_{j}=a_{j}$ for every idempotent $e$;

(3.6) $\quad(\alpha-\beta)^{0}{ }_{i}=\left(\alpha_{i j} \beta_{i j}+a_{j}\right) a_{i}$;

(3.7) $\alpha_{k j} \leqslant(\alpha-\beta)_{k j}+\beta^{r}{ }_{j}$.

Proof of (3.3). $\alpha \beta=0$ means

$$
a_{i}=\left(\alpha_{i j}+\beta_{j k}\right)\left(a_{i}+a_{k}\right) .
$$

Because of the indivisibility of inverses ${ }^{3}$, this is equivalent to each of

$$
\begin{gathered}
a_{i} \leqslant\left(\alpha_{i j}+\beta_{j k}\right)\left(a_{i}+a_{k}\right), \\
a_{i} \leqslant \alpha_{i j}+\beta_{j k}, \\
a_{i}+\alpha_{i j} \leqslant \alpha_{i j}+\beta_{j k}, \\
\left(a_{i}+\alpha_{i j}\right)\left(a_{j}+a_{k}\right) \leqslant\left(\alpha_{i j}+\beta_{j k}\right)\left(a_{j}+a_{k}\right) .
\end{gathered}
$$

(Add $\alpha_{i j}$ to both sides of (3.9) to derive (3.8).) Thus $\alpha \beta=0$ is equivalent to each of :

$$
\left(a_{i}+\alpha_{i j}\right) a_{j} \leqslant \beta_{j k}+\alpha_{i j}\left(a_{j}+a_{k}\right), \alpha^{r}{ }_{j} \leqslant \beta_{j k}, \alpha^{r}{ }_{j} \leqslant a_{j} \beta_{j k}=\beta^{0}{ }_{j} .
$$

Proof of (3.4). That $(1-e)^{r}{ }_{j} \leqslant e^{0}{ }_{j}$ follows from (3.3). Let $\alpha$ be an $L$ number with $\alpha^{r}{ }_{j}=\left[e^{0}{ }_{j}-(1-e)^{r}{ }_{j}\right]$. Then $\alpha^{r}{ }_{j} \leqslant e^{0}{ }_{j}$; by (3.3) this implies $\alpha e=0$, hence $\alpha(1-e)=\alpha$, hence, by $(3.2), \alpha^{r}{ }_{j} \leqslant(1-e)^{r}{ }_{j}$. Since $\alpha^{r}{ }_{j}(1-e)^{r}{ }_{j}$ $=0$ (from the definition of $\alpha$ ) this implies $\alpha^{r}{ }_{j}=0$ and hence $e^{0}{ }_{j}=(1-e)^{r}{ }_{j}$.

${ }^{3}$ The indivisibility of inverses, which follows from the modular law, states: if $y_{1}$ and $y_{2}$ are both inverses of $a$ in $b$ and $y_{1} \leqslant y_{2}$, then $y_{1}=y_{2}$. Because of this, "points" as used in certain constructions in the classical theory of projective geometry may be replaced by "inverses", (1, Section 2.4; 5, Lemma 3.3, p. 32). 
Proof of (3.5). Because of (3.1) and (3.2), the correspondence $(\alpha)_{l} \leftrightarrow \alpha^{r}{ }_{j}$ is a $(1,1)$ order preserving correspondence between the left principal ideals of $\Re$ and the $x \leqslant L\left(a_{j}\right)$; it follows that $e^{r}{ }_{j} \oplus(1-e)^{r}{ }_{j}=a_{j}$. Now (3.5) follows from (3.4).

Proof of (3.6). Using (1.3),

$$
\begin{aligned}
(\alpha-\beta)_{k}{ }_{k} & =\left[\alpha_{i j}+\left(a_{k}+\beta_{i j}\right)\left(a_{j}+c_{i k}\right)\right] a_{k} \\
& =\left[\alpha_{i j}\left(a_{k}+\beta_{i j}\right)+\left(a_{k}+\beta_{i j}\right)\left(a_{j}+c_{i k}\right)\right] a_{k} \\
& =\left[\alpha_{i j} \beta_{i j}+\left(a_{j}+c_{i k}\right)\left(a_{k}+\beta_{i j}\right)\right] a_{k} \\
& =\left(\alpha_{i j} \beta_{i j}+a_{j}+c_{i k}\right)\left(a_{k}+\beta_{i j}\right) a_{k} \\
& =\left(\alpha_{i j} \beta_{i j}+a_{j}+c_{i k}\right) a_{k} .
\end{aligned}
$$

Hence

$$
\begin{aligned}
(\alpha-\beta)^{0}{ }_{i} & =\left[(\alpha-\beta){ }^{0}{ }_{k}+c_{i k}\right] a_{i} \\
& =\left(\alpha_{i j} \beta_{i j}+a_{j}+c_{i k}\right)\left(a_{k}+c_{i k}\right) a_{i} \\
& =\left(\alpha_{i j} \beta_{i j}+a_{j}\right) a_{i} .
\end{aligned}
$$

Proof of (3.7). Using (1.3),

$$
\begin{aligned}
(\alpha-\beta)_{k j}+\beta^{r}{ }_{j} & =\left[\alpha_{i j}+\left(a_{k}+\beta_{i j}\right)\left(a_{j}+c_{i k}\right)\right]\left(a_{k}+a_{j}\right)+\left(\beta_{i j}+a_{i}\right) a_{j} \\
& =\left[\alpha_{i j}+\left(\beta_{i j}+a_{i}\right) a_{j}+\left(a_{k}+\beta_{i j}\right)\left(a_{j}+c_{i k}\right)\right]\left(a_{k}+a_{j}\right) \\
& =\left[\alpha_{i j}+\left(\beta_{i j}+a_{i}+a_{k}\right) a_{j}+\left(a_{k}+\beta_{i j}\right)\left(a_{j}+c_{i k}\right)\right]\left(a_{k}+a_{j}\right) \\
& =\left[\alpha_{i j}+\left(\beta_{i j}+a_{i}+a_{k}\right)\left(a_{j}+a_{k}+\beta_{i j}\right)\left(a_{j}+c_{i k}\right)\right]\left(a_{k}+a_{j}\right) \\
& \geqslant\left(\alpha_{i j}+c_{i k}\right)\left(a_{k}+a_{j}\right)=\alpha_{k j} .
\end{aligned}
$$

4. The $i$-elements in $L$. We shall call $x$ an $i$-element if $x \leqslant A^{i}$ and $x A^{i-1}=0$. We shall prove that for arbitrary $L$-numbers $\beta^{j}(j=1, \ldots i-1)$ and arbitrary idempotent $e$, the formula:

$$
x=\left(e_{i}+A^{i-1}\right) \prod_{j=1}^{i-1}\left(\beta_{i j}^{j}+A_{j}^{i-1}\right)
$$

defines an $i$-element, (in particular, if $e=1, e^{r}{ }_{i}+A^{i-1}=a_{i}+A^{i-1}$ and may be omitted). For clearly $x \leqslant A^{i}$ and

$$
\begin{aligned}
x A^{i-1} & =A^{i-1} \prod_{j=1}^{i-1}\left(\beta^{j}{ }_{i j}+A^{i-1}{ }_{j}\right) \\
& =A^{i-1} \prod_{j=1}^{i-1}\left(\beta^{j}{ }_{i j} a_{j}+A^{i-1}{ }_{j}\right) \\
& =\prod_{j=1}^{i-1} A^{i-1}{ }_{j}=0 .
\end{aligned}
$$

For each $i$-element $x$ we define:

$$
x^{j}=\left(x+A^{i-1}{ }_{j}\right)\left(a_{i}+a_{j}\right), \quad j=1, \ldots, i-1 .
$$

For each choice of idempotent $e=e(x)$ with $e^{r}{ }_{i}=\left(x+A^{i-1}\right) a_{i}$ (such an $e$ exists by (3.1)), we define:

$$
B=B(x)=x+e^{0}{ }_{i}, \quad B^{j}=\left(B+A^{i-1}{ }_{j}\right)\left(a_{i}+a_{j}\right) .
$$

( $B$ may not be uniquely determined by $x$.) The following relations hold: 
(4.4) $e^{\tau}{ }_{i}+A^{i-1}=x+A^{i-1}, e^{r}{ }_{i}+a_{j}=x^{j}+a_{j}, x^{j}+A^{i-1}{ }_{j}=x+A^{i-1}{ }_{j}$,

(4.5) $B A^{i-1}=\left[x+e^{0}{ }_{i}\left(x+A^{i-1}\right)\right] A^{i-1}=\left(x+e^{0} e^{r}{ }_{i}\right) A^{i-1}=0$, by (3.5), $B+A^{i-1}=x+A^{i-1}+e^{0}{ }_{i}=x+A^{i-1}+e^{r}{ }_{i}+e^{0}{ }_{i}$ $=x+A^{i-1}+a_{i}=A^{i}$.

(4.6) $B^{j} a_{j}=\left(B+A^{i-1}{ }_{j}\right) a_{j}=\left(B A^{i-1}+A^{i-1}{ }_{j}\right) a_{j}=A^{i-1}{ }_{j} a_{j}=0$, $B^{j}+a_{j}=\left(B+A^{i-1}{ }_{j}+a_{j}\right)\left(a_{i}+a_{j}\right)=\left(B+A^{i-1}\right)\left(a_{i}+a_{j}\right)$ $=A^{i}\left(a_{i}+a_{j}\right)=a_{i}+a_{j}$.

(4.7) $B\left(e^{r}{ }_{i}+A^{i-1}\right)=B\left(x+A^{i-1}\right)=x+B A^{i-1}=x$, $B^{j}\left(e^{r}{ }_{i}+a_{j}\right)=\left(B+A^{i-1}{ }_{j}\right)\left(x^{j}+a_{j}\right)=x^{j}+\left(B+A^{i-1}{ }_{j}\right) a_{j}=x^{j}$.

$B^{j} a_{i}=\left(x+e^{0}{ }_{i}+A^{i-1}{ }_{j}\right) a_{i}=e^{0}{ }_{i}+\left(x+A^{i-1}{ }_{j}\right) a_{i}$ $=e_{i}^{0}+\left(x^{j}+A^{i-1}{ }_{j}\right) a_{i}$.

Thus $B^{j}$ is in $L_{i j}$ for each $j=1, \ldots, i-1$. Let $\alpha^{j}$ be the $L$-number with $\alpha^{j}{ }_{i j}=B^{j}$. Then

$$
\alpha^{j}{ }_{i j} \geqslant x^{j}, \quad e \alpha^{j}=\alpha^{j}=e \beta^{j}
$$

for every $L$-number $\beta^{j}$ with $\beta^{j}{ }_{i j} \geqslant x^{j}$. To verify (4.9): from $(4.7), \alpha^{j}{ }_{i j}=B^{j} \geqslant x^{j}$; from (4.8), $\left(a^{j}\right)^{0}{ }_{i}=B^{j} a_{i} \geqslant e^{0}{ }_{i}=(1-e)^{r}{ }_{i}$ and (3.3) then implies $(1-e) \alpha^{j}=0$, i.e., $e \alpha^{j}=\alpha^{j}$; finally, from (3.6),

$$
\left(\alpha^{j}-\beta^{j}\right)_{i}^{0}=\left(B^{j} \beta^{j}{ }_{i j}+a_{j}\right) a_{i} \geqslant\left(B^{j} x^{j}+a_{j}\right) a_{i}=\left(e^{r}{ }_{i}+a_{j}\right) a_{i}=e^{r}{ }_{i}
$$

and by (3.3), $e\left(\alpha^{j}-\beta^{j}\right)=0$, that is, $e \alpha^{j}=\alpha^{j}=e \beta^{j}$.

Suppose $\bar{e}$ is also a possible choice of $e(x)$ and let $\bar{\alpha}^{j}(j=1, \ldots, i-1)$ denote the corresponding $L$-numbers. The definition of $e(x)$ shows that $\bar{e}^{r}=e^{r}$ and then (3.2) implies $\bar{e} e=\bar{e}, e \bar{e}=e$. Now (4.9) implies

$$
\bar{e} \alpha^{j}=\bar{\alpha}^{j},
$$$$
1 \leqslant j<i
$$

The following relations follow easily from (4.3) and (4.7):

$$
\begin{aligned}
& B=\prod_{j=1}^{i-1}\left(\alpha^{j}{ }_{i j}+A_{j}^{i-1}\right) \\
& x=\left(e^{r}{ }_{i}+A^{i-1}\right) \prod_{j=1}^{i-1}\left(\alpha^{j}{ }_{i j}+A_{j}^{i-1}\right) .
\end{aligned}
$$

We shall prove that for arbitrary $\beta^{j}$ with $\beta^{j}{ }_{i j} \geqslant x^{j}$ for $j=1, \ldots, i-1$, the $i$-element:

$$
\left(e^{r}{ }_{i}+A^{i-1}\right) \prod_{j=1}^{i-1}\left(\beta^{j}{ }_{i j}+A^{i-1}{ }_{j}\right)
$$

is identical with $x$. To prove this let (4.12) be denoted by $y$. Then

$$
\begin{aligned}
\left(e_{i}^{r}+A^{i-1}\right)\left(\alpha_{i j}^{j}+A_{j}^{i-1}\right) & =A^{i-1}{ }_{j}+B^{j}\left(e^{r}{ }_{i}+A^{i-1}\right)\left(a_{i}+a_{j}\right) \\
& =A^{i-1}{ }_{j}+B^{j}\left(e^{r}{ }_{i}+a_{j}\right)=A^{i-1}{ }_{j}+x^{j} \\
\leqslant & A^{i-1}{ }_{j}+\beta^{j}{ }_{i j}
\end{aligned}
$$

for each $j=1, \ldots, i-1$, and hence 


$$
\begin{aligned}
& x=\left(e^{r}+A^{i-1}\right) \prod_{j=1}^{i-1}\left(\alpha_{i j}^{j}+A^{i-1}{ }_{j}\right) \\
& \leqslant\left(e_{i}^{r}+A^{i-1}\right) \prod_{j=1}^{i-1}\left(\beta^{j}{ }_{i j}+A^{i-1}{ }_{j}\right)=y,
\end{aligned}
$$

that is, $x \leqslant y$. But since $x$ and $y$ are both $i$-elements, it follows from the indivisibility of inverses that $x=y$. (For $y+A^{i-1}=x+\mathrm{A}^{i-1}$, and hence $y=y\left(x+A^{i-1}\right)=x+y A^{i-1}=x$.)

Conversely, for arbitrary idempotent $e$ and arbitrary $L$-numbers $\beta^{j}$, the formula (4.12) defines an element $x$ in $L$ with the properties: $x$ is an $i$-element, $x^{j} \leqslant \beta^{j}{ }_{i j}$ for $j=1, \ldots, i-1$, and $e$ is a possible choice for $e(x)$. For, $\left(x+A^{i-1}\right) a_{i}=e^{r}{ }_{i}$ and

$$
\begin{aligned}
x^{j} & =\left(x+A_{j}^{i-1}\right)\left(a_{i}+a_{j}\right) \\
& =\left[\left(e^{r}{ }_{i}+A^{i-1}\right) \prod_{k=1}^{i-1}\left(\beta_{i k}^{k}+A^{i-1}\right)+A^{i-1}{ }_{j}\right]\left(a_{i}+a_{j}\right) \\
& =\left(e^{r}{ }_{i}+A^{i-1}\right)\left(\beta^{j}{ }_{i j}+A^{i-1}{ }_{j}\right)\left(a_{i}+a_{j}\right) \\
& =\left[\left(e^{r}{ }_{i}+a_{j}\right) \beta^{j}{ }_{i j}+A^{i-1}{ }_{j}\right]\left(a_{i}+a_{j}\right)=\left(e^{r}+a_{j}\right) \beta^{j}{ }_{i j} \\
& \leqslant \beta^{j}{ }_{i j},
\end{aligned}
$$

as stated. Moreover, (4.9) shows that $\alpha^{j}(x)=e \beta^{j}$.

5. An important identity. Suppose that $1 \leqslant j<n, i=j+1$, and let $\delta^{m}$ and $\theta^{m}, m=1, \ldots, j$, and $\beta$ be arbitrary elements in $\Re$. Then the following identity holds: ${ }^{4}$

$$
\begin{aligned}
& \prod_{m=1}^{j-1}\left[\left(\delta^{m}+\beta \theta^{m}\right)_{i m}+A_{m}^{j-1}\right] \\
& =\left[\left(\beta_{i j}+A^{j-1}\right) \prod_{m=1}^{j-1}\left(\delta^{m}{ }_{i m}+A_{m}^{j}\right)+\prod_{m=1}^{j-1}\left(\theta^{m}{ }_{j m}+A_{m}^{j-1}\right)\right]\left(A^{j-1}+a_{i}\right) .
\end{aligned}
$$

To prove this identity we shall first establish that for arbitrary indices $i$, $j, m$ all different, and arbitrary $\delta, \beta, \theta$ in $\Re$,

$$
(\delta+\beta \theta)_{i m}=\left[\theta_{j m}+\left(\delta_{i m}+a_{j}\right)\left(\beta_{i j}+a_{m}\right)\right]\left(a_{i}+a_{m}\right) .
$$

Indeed the addition formula (2.1) shows that if $k$ is different from $i, j, m$, (and there is such a $k$ since we assume $n \geqslant 4$ ),

${ }^{4}$ This identity makes possible a simple proof that the module which we shall assign to an $x$ in $L$ (see section 6), is uniquely determined by $x$ (Theorem 6.5 ). This is a critical step in the proof of the coordinatization theorem as given in the present paper. It will be noted that the proof of this identity uses the same technique that was used by one of us in 1937 to obtain a direct proof of the associativity and distributivity laws in $\Re(1, \S \S 4.11,4.12)$. 


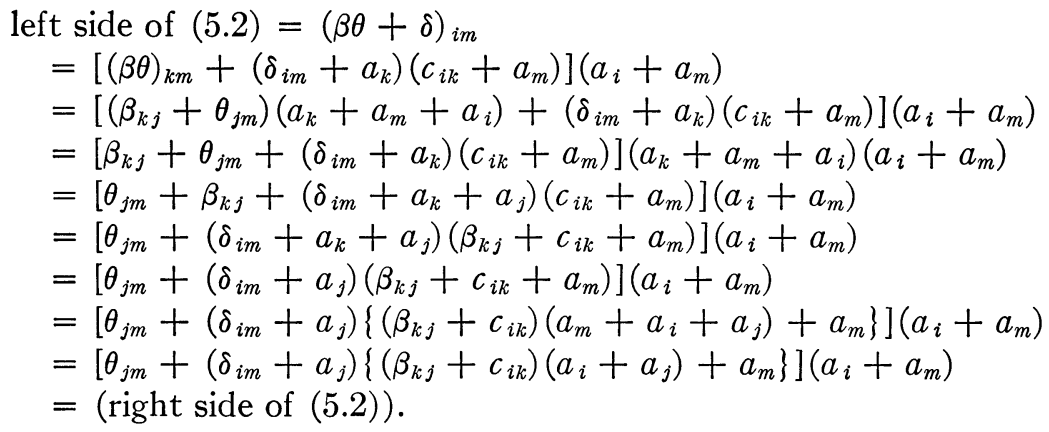

This proves (5.2). It follows that

$$
\begin{aligned}
\text { (left side of }(5.1)) & =\prod_{m=1}^{j-1}\left[\left\{\theta^{m}{ }_{j m}+\left(\delta^{m}{ }_{i m}+a_{j}\right)\left(\beta_{i j}+a_{m}\right)\right\}\left(a_{i}+a_{m}\right)+A^{j-1}\right] \\
& =\left(a_{i}+A^{j-1}\right) \prod\left[\left(\theta^{m}{ }_{j m}+A^{j-1}{ }_{m}\right)+\left(\delta^{m}{ }_{i m}+A_{m}^{j}\right)\left(\beta_{i j}+A^{j-1}\right)\right] \\
& \geqslant \text { (right side of }(5.1)) .
\end{aligned}
$$

But both the left side and the right side of (5.1) are inverses of $A^{j-1}$ in $a_{i}+A^{j-1}$; for the left side of (5.1) is a j-element and hence:

$A^{j-1}$ (right side of $\left.(5.1)\right) \leqslant A^{j-1}$ (left side of (5.1)) $=0$, and

$$
\begin{aligned}
a_{i}+A^{j-1} \geqslant & \text { (left side of }(5.1))+A^{j-1} \\
\geqslant & \text { (right side of }(5.1))+A^{j-1} \\
= & \left(a_{i}+A^{j-1}\right)\left[\prod_{m=1}^{j-1}\left(\theta^{m}{ }_{j m}+A^{j-1}{ }_{m}\right)\right. \\
& \left.\quad+\left(\beta_{i j}+A^{j-1}\right) \prod_{m=1}^{j-1}\left(\delta^{m}{ }_{i m}+A^{j}{ }_{m}\right)+A^{j-1}\right] \\
& =\left(a_{i}+A^{j-1}\right)\left[\prod\left(\theta^{m}{ }_{j m}+A_{m}^{j-1}\right)+\left(\beta_{i j}+A^{j-1}\right) \prod\left(\delta^{m}{ }_{i m}+A^{j}\right)\right] \\
& =\left(a_{i}+A^{j-1}\right)\left[\prod\left(\theta^{m}{ }_{j m}+A_{m}^{j-1}\right)+\left(\beta_{i j}+A^{j-1}\right) \prod\left(a_{i}+A^{j}\right)\right] \\
& =\left(a_{i}+A^{j-1}\right)\left[\prod\left(\theta^{m}{ }_{j m}+A_{m}^{j-1}\right)+\beta_{i j}+A^{j-1}\right] \\
& =\left(a_{i}+A^{j-1}\right)\left[\prod\left(\theta^{m}{ }_{j m}+A_{m}^{j-1}+a_{m}\right)+\beta_{i j}\right] \\
& =\left(a_{i}+A^{j-1}\right)\left[\prod\left(A^{j}\right)+\beta_{i j}\right] \\
& =\left(a_{i}+A^{j-1}\right)\left(a_{i}+A^{j}\right) \\
& =\left(a_{i}+A^{j-1}\right) .
\end{aligned}
$$

Now the indivisibility of inverses shows that equality holds in (5.1).

${ }^{5}$ (ST) will indicate application of the superfluous term identity: $a b=a(b+c)=a b c$ for all $a, b, c, d$ with $c(a+b)=0$ and $b \leqslant d$; similarly, for application of the modular law we use (ML); for the absorption law: $a b+c=a(b+c)$ for all $a, b, c$ with $c \leqslant a$ we use (AL); and for the clipping identity $a(b+c)=a[b(a+c)+c]$ for all $a, b, c$ we use (CI) (1, Section 2.2). 
As a corollary to (5.1) we shall derive the following identity which holds for arbitrary $\beta^{m}$ and $\theta^{m}, m=1, \ldots, j, i=j+1$, and arbitrary $\gamma$ :

$$
\begin{gathered}
{\left[\left(\beta^{j}-\gamma\right)_{i j}+A^{j-1}\right] \prod_{m=1}^{j-1}\left[\left(\beta^{m}+\gamma \theta^{m}\right)_{i m}+A_{m}^{j}\right]+\prod_{m=1}^{j-1}\left(\theta^{m}{ }_{j m}+A_{m}^{j-1}\right)} \\
=\left(\beta_{i j}^{j}+A^{j-1}\right) \prod_{m=1}^{j-1}\left(\beta^{m}{ }_{i m}+A_{m}^{j}\right)+\prod_{m=1}^{j-1}\left(\theta^{m}{ }_{j m}+A_{m}^{j-1}\right) .
\end{gathered}
$$

The right side of (5.3) is precisely the left side of (5.3) with a particular value for $\gamma, \gamma=0$. Thus we need only show that for any $\gamma$ the left side of (5.3) has the same value, equal to its value when $\gamma=\beta^{j}$, say. For this purpose it is sufficient to establish the identity:

(5.4) (left side of (5.3)) $=\prod\left[\left(\beta^{m}+\beta^{j} \theta^{m}\right)_{i m}+A_{m}^{j-1}\right]+\prod\left(\theta_{j m}^{m}+A_{m}^{j-1}\right)$.

Now (5.4) can be obtained by substituting in (5.1): $\beta^{j}-\gamma$ for $\beta$ and $\beta^{m}+\gamma \theta^{m}$ for $\delta^{m}, m=1, \ldots, j-1$ and adding the term $\Pi\left(\theta^{m}{ }_{j m}+A^{j-1}{ }_{m}\right)$ to both sides.

6. The rule for assigning left modules to elements of $\boldsymbol{L}$. For each $x$ in $L$ call $x_{1}, \ldots, x_{n}$ a base-decomposition of $x$ if each $x_{i}$ is an inverse:

$$
x_{i}=\left[x A^{i}-x A^{i-1}\right], \quad i=1, \ldots, n .
$$

Clearly each $x_{i}$ is an $i$-element and $x=x_{1} \oplus \ldots \oplus x_{n}$. For each base-decomposition of $x$ and for any idempotent $e^{i}$ satisfying:

$$
\left(e^{i}\right)^{r}{ }_{i}=\left(x_{i}+A^{i-1}\right) a_{i}=\left(x A^{i}+A^{i-1}\right) a_{i},
$$

let $B\left(x_{i}\right), B^{j}\left(x_{i}\right), \alpha^{i j}=\alpha^{j}\left(x_{i}\right)$ be determined as in $\$ 4$, and define the vector

$$
u\left(x_{i}\right)=\left(-\alpha^{i 1}, \ldots,-\alpha^{i, i-1}, e^{i}, 0, \ldots, 0\right) .
$$

Now, for each such $u\left(x_{i}\right), i=i, \ldots, n$, assign to $x$ the left module

$$
M\left(x_{1}, \ldots, x_{n}\right)=\left(u\left(x_{1}\right), \ldots, u\left(x_{n}\right)\right)_{l} .
$$

We note that: (i) the $x_{i}$ may not be uniquely determined by $x$ and for each $x_{i}$ the idempotent $e^{i}$ may not be uniquely determined by $x_{i}$, however it follows from $(4.9)^{\prime}$ that $\left(u\left(x_{i}\right)\right)_{l}$ is uniquely determined by $x_{i}$ so that $M\left(x_{1}, \ldots, x_{n}\right)$ is uniquely determined by $x_{1}, \ldots, x_{n}$; (ii) if $x$ is a $j$-element then the $x_{i}$ are uniquely determined with $x_{i}=x$ for $i=j$ and $x_{i}=0$ for $i \neq j$; (iii) if $x_{i}$ is an arbitrary $i$-element for each $i=1, \ldots, n$, and $x=x_{1}+\ldots+x_{n}$ then $x_{1}, \ldots, x_{n}$ is a base-decomposition for $x$.

We shall prove below the following statements (6.1)-(6.7).

(6.1) Every left module $M$ of finite span is identical with $M\left(x_{1}, \ldots, x_{n}\right)$ for some base-decomposition $x_{1}, \ldots, x_{n}$ of some $x$ in $L$. 
(6.2) Suppose $x_{1}, \ldots, x_{n}$ and $\bar{x}_{1}, \ldots, \bar{x}_{n}$ are base-decompositions for the same $x$. If

$$
u\left(x_{m}\right)=\left(-\alpha^{m, 1}, \ldots,-\alpha^{m, m-1}, e^{m}, 0, \ldots, 0\right)
$$

and

$$
u\left(\bar{x}_{m}\right)=\left(-\bar{\alpha}^{m, 1}, \ldots,-\bar{\alpha}^{m, m-1}, \bar{e}^{m}, 0, \ldots, 0\right)
$$

and the $\alpha^{m, k}$ and the $\bar{\alpha}^{m, k}$ both form canonical matrices ${ }^{6}$ with $\bar{e}^{m}=e^{m}$, then $M\left(x_{1}, \ldots, x_{n}\right)=M\left(\bar{x}_{1}, \ldots, \bar{x}_{n}\right)$.

(6.3). Suppose $y$ is an $i$-element and $z$ is a $j$-element with $1 \leqslant j<i \leqslant n$. If for some $\gamma$ in $\Re$ there is a relation $u(x)=u(y)+\gamma u(z)$ for some $i$-element $x$, then $x \leqslant y+z$; on the other hand if $e$ is the $i$ th coordinate of $u(y)$ and $\gamma$ satisfies $e \gamma=\gamma$ then the vector $u(y)+\gamma u(z)$ has $i$ th coordinate $=e$ and coincides with $u(x)$ for some $i$-element $x$ with $z+x=z+y$.

(6.4). For each base-decomposition $x_{1}, \ldots, x_{n}$ of $x$ and choice of the $u\left(x_{m}\right)$ there exists a base-decomposition $\bar{x}_{1}, \ldots, \bar{x}_{n}$ of $x$ and choices of $u\left(\bar{x}_{m}\right)$ :

$$
u\left(\bar{x}_{m}\right)=\left(-\bar{\alpha}^{m, 1}, \ldots,-\bar{\alpha}^{m, m-1}, e^{m}, 0, \ldots, 0\right)
$$

such that

$(6.4)^{\prime} M\left(\bar{x}_{1}, \ldots, \bar{x}_{n}\right)=M\left(x_{1}, \ldots, x_{n}\right)$,

$(6.4)^{\prime \prime} \quad \bar{\alpha}^{m, k} e^{k}=0$

for all $n \geqslant m>k \geqslant 1$,

implying that the $\bar{\alpha}^{m, k}$ form a canonical matrix.

(6.5) For each $x$ in $L$, all $M\left(x_{1}, \ldots, x_{n}\right)$ assigned to $x$ coincide, so that we may write $M(x)$ for $M\left(x_{1}, \ldots, x_{n}\right)$.

(6.6) $x \leqslant y$ implies that $M(x) \leqslant M(y)$.

(6.7) $M(x) \leqslant M(y)$ implies $x \leqslant y$.

The coordinatization theorem follows easily from (6.1), (6.5), (6.6) and (6.7).

7. Proof of (6.1). $M$ is spanned by some canonical basis $u^{i}=\left(\alpha^{i 1}, \ldots, \alpha^{i n}\right)$, $i=1, \ldots, n(\mathbf{1}, \S 3.4)$. Choose $e^{i}, x(i)$ as follows:

$$
\begin{aligned}
e^{i} & =\alpha^{i i}, \\
x(i) & =\left[\left(e^{i}\right)^{r}{ }_{i}+A^{i-1}\right] \prod_{j=1}^{i-1}\left[\left(-\alpha^{i j}\right)_{i j}+A_{j}^{i-1}\right] .
\end{aligned}
$$

\footnotetext{
${ }^{6}$ It has been shown $(5$, Theorem 2.12 and Lemma $2.11 ; 1$, Section 3.4$)$ that a left module of finite span is always spanned by $n$ vectors $\left(\alpha^{j 1}, \ldots, \alpha^{j n}\right), j=1, \ldots, n$, with the properties: for each $j, \alpha^{i j}$ is idempotent, $=e^{i}$, say; for all $i>j, \alpha^{i i}=0$; for all $i<j, e^{i} \alpha^{j i}=\alpha^{j i}$ and $\alpha^{j i} e^{i}=0$. Such a set of $n$ vectors is called a canonical basis for the left module. A matrix whose rows form such a canonical basis is called a canonical matrix.
} 
Then $\S 4$ shows that each $x(i)$ is an $i$-element and that $e^{i}$ is a possible choice for $e(x(i))$; with this choice of $e(x(i))$ it follows from the last paragraph of $\S 4$ that $\alpha^{j}(x(i))=-\alpha^{i j}$, (for a canonical basis $e^{i} \alpha^{i j}=\alpha^{i j}$ ), that $u^{i}$ is a possible choice for $u(x(i))$ and hence $M$ coincides with $M\left(x_{1}, \ldots, x_{n}\right)$.

8. Proof of (6.2). We shall show that $\bar{x}_{m}=x_{m}$ for all $m$ so that the $\alpha^{m k}$ are uniquely determined by $x$ and the $e^{m}$ (if the $\alpha^{m k}$ are to form a canonical matrix).

Set $U^{k}=\left(e^{k}\right)^{0}{ }_{k} \leqslant a_{k}$ for each $k<m$. Since (3.5) shows that $\left(e^{k}\right)^{0}{ }_{k}\left(e^{k}\right)^{r}{ }_{k}=0$, it follows that

$$
U^{k}\left(x_{k}+A^{k-1}\right)=0 .
$$

We shall show that

$$
x_{m}=x \prod_{k=1}^{m-1}\left(U^{k}+A_{k}^{m}\right) ;
$$

this will establish the uniqueness of $x_{m}$ since the $U^{k}$ are uniquely determined by the $e^{k}$.

From $\$ 4$ there is a $B\left(x_{m}\right) \geqslant x_{m}$ for which

$$
\alpha_{m k}^{m k}=\left(B\left(x_{m}\right)+A_{k-1}^{m}\right)\left(a_{m}+a_{k}\right) ;
$$

now (3.3) implies that $\left(\alpha^{m k}\right)^{r}{ }_{k} \leqslant\left(e^{k}\right)^{0}{ }_{k}$; i.e., $\left(B\left(x_{m}\right)+A^{m}{ }_{k}\right) a_{k} \leqslant U^{k}$. Hence $U^{k}+A_{k}^{m} \geqslant B\left(x_{m}\right) \geqslant x_{m}$ for each $k$ and so (right side of $\left.(8.2)\right) \geqslant x_{m}$. Now

(right side of $(8.2))=x A^{m} \prod_{k=1}^{m-1}\left(U^{k}+A_{k}^{m}\right)=\left(x_{1}+\ldots+x_{m}\right) \prod_{k=1}^{m-1}\left(U^{k}+A_{k}^{m}\right)$

$$
=x_{m}+\left(x_{1}+\ldots+x_{m-1}\right) \prod_{k=1}^{m-1}\left(U^{k}+A_{k}^{m}\right) \text {. }
$$

But

$$
\begin{aligned}
\left(x_{1}+\ldots\right. & \left.+x_{m-1}\right) \prod_{k=1}^{m-1}\left(U^{k}+A_{k}^{m}\right)=\left(x_{1}+\ldots+x_{m-1}\right) \prod_{k=1}^{m-1}\left(U^{k}+A_{k}^{m-1}\right) \\
& =\left(x_{1}+\ldots+x_{m-1}\right)\left(U^{m-1}+A^{m-2}\right) \prod_{k=1}^{m-2}\left(U^{k}+A_{k}^{m-1}\right) \\
& =\left(x_{1}+\ldots+x_{m-2}\right) \prod_{k=1}^{m-2}\left(U^{k}+A_{k}^{m-1}\right)
\end{aligned}
$$

since (8.1) and (CI) show that $x_{m-1}\left(U^{m-1}+A^{m-2}\right)=0$. Repetition of the reduction in (8.4) shows that the second addend of (8.3) is zero and establishes (8.2).

9. Proof of (6.3). If $e$ is the $i$ th coordinate of $u(y)$ with $y$ an $i$-element and $z$ a $j$-element, $1 \leqslant j<i \leqslant n$, and $\gamma$ is an element of $\Re$ with $e \gamma=\gamma$, then $u(y)+\gamma u(z)$ is a vector of the form:

$$
\left(-e \beta^{1},-e \beta^{2}, \ldots,-e \beta^{i-1}, e, 0, \ldots, 0\right),
$$

and hence coincides with $u(x)$ for some $i$-element $x$, by the last paragraph of $\S 4$. This proves the second part of (6.3) except for the relation $z+x=z+y$. 
However this relation follows easily from the first part of (6.3), which we now prove.

Because of $\$ 4$ the first part of $(6.3)$ is equivalent to the statement:

$$
\begin{gathered}
\left(\bar{e}_{i}^{r}+A^{i-1}\right) \prod_{m=1}^{i-1}\left(\alpha^{m}{ }_{i m}+A^{i-1}{ }_{m}\right) \\
\leqslant\left(\bar{e}_{i}^{r}+A^{i-1}\right) \prod_{m=1}^{i-1}\left(\beta^{m}{ }_{i m}+A_{m}^{i-1}\right)+\left(e_{j}^{r}+A^{j-1}\right) \prod_{m=1}^{j-1}\left(\theta^{m}{ }_{j m}+A_{m}^{j-1}\right)
\end{gathered}
$$

for arbitrary idempotents $\bar{e}$ and $e$ in $\Re$ provided that $\bar{e} \alpha^{m}=\alpha^{m}$ for $m<i$, $\bar{e} \beta^{m}=\beta^{m}$ for $m<i, e \theta^{m}=\theta^{m}$ for $m<j$ and

$$
\left\{\begin{array}{lr}
\alpha^{m}=\beta^{m} & \text { for } j<m \leqslant i, \\
\alpha^{j}=\beta^{j}-\gamma e & \text { for some } \gamma, \\
\alpha^{m}=\beta^{m}+\gamma \theta^{m} & \text { for } 1 \leqslant m<j .
\end{array}\right.
$$

Now (9.1) can be deduced from the (apparently) simpler statement:

$$
\prod_{m=1}^{i-1}\left(\alpha^{m}{ }_{i m}+A^{i-1}{ }_{m}\right) \leqslant \prod_{m=1}^{i-1}\left(\beta^{m}{ }_{i m}+A_{m}^{i-1}\right)+\left(e^{r}+A^{j-1}\right) \prod_{m=1}^{j-1}\left(\theta_{j m}^{m}+A_{m}^{j-1}\right)
$$

for arbitrary idempotent $e$ and arbitrary $\theta^{m}$ satisfying $e \theta^{m}=\theta^{m}$ for $m<j$ and arbitrary $\alpha^{m}, \beta^{m}$ satisfying $(9.1)^{\prime}$ for some $\gamma$. Indeed, clipping (9.2) by $\bar{e}^{r}{ }_{i}+A^{i-1}$ yields (9.1) as required.

If $j<i-1$ then $\alpha^{m}=\beta^{m}$ for $j+1 \leqslant m \leqslant i-1$ and (9.2) can be deduced from:

(9.3) $\prod_{m=1}^{j}\left(\alpha^{m}{ }_{i m}+A^{i-1}{ }_{m}\right) \leqslant \prod_{m=1}^{j}\left(\beta^{m}{ }_{i m}+A^{i-1}{ }_{m}\right)+\left(e^{r}+A^{j-1}\right) \prod_{m=1}^{j-1}\left(\theta^{m}{ }_{j m}+A^{j-1}{ }_{m}\right)$;

for clipping (9.3) by

$$
\prod_{m=j+1}^{i-1}\left(\alpha^{m}{ }_{i m}+A_{m}^{i-1}\right)
$$

gives (9.2).

Since $a_{1}, \ldots, a_{i}$ are independent, we may write $(9.3)$ (use $(\mathbf{1}, \S 2.3)$ ) as:

$$
\left(a_{j+1}+\ldots+a_{i-1}\right)+\prod_{m=1}^{j}\left(\alpha^{m}{ }_{i m}+A_{m}^{j}\right)
$$

$\leqslant\left(a_{j+1}+\ldots+a_{i-1}\right)+\prod_{m=1}^{j}\left(\beta^{m}{ }_{i m}+A_{m}^{j}\right)+\left(e_{j}^{r}+A^{j-1}\right) \prod_{m=1}^{j-1}\left(\theta^{m}{ }_{j m}+A^{j-1}{ }_{m}\right)$

and this would clearly be a consequence of

$$
\text { (9.5) } \prod_{m=1}^{j}\left(\alpha^{m}{ }_{i m}+A_{m}^{j}\right) \leqslant \prod_{m=1}^{j}\left(\beta^{m}{ }_{i m}+A_{m}^{j}\right)+\left(e_{j}^{\tau}+A^{j-1}\right) \prod_{m=1}^{j-1}\left(\theta^{m}{ }_{j m}+A^{j-1}{ }_{m}\right) .
$$

But (9.5) can be derived from

$$
\prod_{m=1}^{j}\left(\alpha^{m}{ }_{i m}+A_{m}^{j}\right) \leqslant \prod_{m=1}^{j}\left(\beta^{m}{ }_{i m}+A_{m}^{j}\right)+\prod_{m=1}^{j-1}\left(\theta^{m}{ }_{j m}+A_{m}^{j-1}\right)
$$


by clipping both sides of $(9.6)$ by $\beta^{j}{ }_{i j}+e^{r}{ }_{j}+A^{j-1}$ : indeed, this clipping does not change the left side of (9.6) since one of its factors is

$$
\begin{aligned}
\alpha^{j}{ }_{i j}+A^{j-1} & =\left(\beta^{j}-\gamma e\right)_{i j}+A^{j-1} \\
& \leqslant \beta^{j}{ }_{i j}+(-\gamma e)^{r}{ }_{j}+A^{j-1} \\
& \leqslant \beta^{j}{ }_{i j}+e^{r}{ }_{j}+A^{j-1}
\end{aligned}
$$

on the other hand this clipping changes the right side of (9.6) to

$$
\begin{aligned}
\prod_{m=1}^{j}\left(\beta^{m}{ }_{i m}\right. & \left.+A^{j}{ }_{m}\right)+\left(\beta^{j}{ }_{i j}+e^{r}{ }_{j}+A^{j-1}\right) \prod_{m=1}^{j-1}\left(\theta^{m}{ }_{j m}+A^{j-1}{ }_{m}\right) \\
= & \prod_{m=1}^{j}\left(\beta^{m}{ }_{i m}+A_{m}^{j}\right)+\left(e^{r}{ }_{j}+A^{j-1}\right) \prod_{m=1}^{j-1}\left(\theta^{m}{ }_{j m}+A^{j-1}{ }_{m}\right)
\end{aligned}
$$

since the modular law implies $\left(\beta^{j}{ }_{i j}+e^{r}{ }_{j}+A^{j-1}\right) A^{j}=e^{r}{ }_{j}+A^{j-1}$.

But since $\alpha^{m}=\beta^{m}+\gamma \theta^{m}$ for $m<j$ and $\alpha^{j}=\beta^{j}-\gamma$, the desired (9.6) follows immediatiely from (5.3) proved above.

10. Proof of (6.4). By (6.3) there exist $\bar{x}_{2}, \ldots, \bar{x}_{n}$ with $x_{1}+\bar{x}_{m}=x_{1}+x_{m}$ for all $m$ (so that $x_{1}, \bar{x}_{2}, \ldots, \bar{x}_{n}$ is again a base-decomposition of $x$ ) and with $u\left(\bar{x}_{m}\right)=u\left(x_{m}\right)+\alpha^{m, 1} u\left(x_{1}\right)$ so that $\bar{\alpha}^{m, 1} e^{1}=0$ for $m>1$. Similarly $\bar{x}_{3}, \ldots, \bar{x}_{n}$ can be replaced so that the new $\bar{\alpha}^{i, j}$ satisfy also $\bar{\alpha}^{m, 2} e^{2}=0$ for $m>2$. Successive repetition of this procedure establishes (6.4).

11. Proof of (6.5). If $M=M\left(x_{1}, \ldots, x_{n}\right)=\left(u\left(x_{1}\right), \ldots, u\left(x_{n}\right)\right)_{l}$ we may, without changing $M$, replace $u\left(x_{m}\right)$ by $e^{m} u\left(x_{m}\right)$ where $e^{m}$ is any idempotent satisfying $\left(e^{m}\right)_{m}^{r}=\left(x A^{m}+A^{m-1}\right) a_{m}$. The statement (6.5) now follows from (6.4) and (6.2).

12. Proof of (6.6). If $x \leqslant y$ then we may choose the $x_{i}, y_{i}$ so that $x_{i} \leqslant y_{i}$ (for example, choose $y_{i}=x_{i}+\left[y A^{i}-\left(y A^{i-1}+x_{i}\right)\right]$. Then $\left(e\left(x_{i}\right)\right)^{r}{ }_{i} \leqslant\left(e\left(y_{i}\right)\right)^{r}{ }_{i}$ which implies $e\left(x_{i}\right) e\left(y_{i}\right)=e\left(x_{i}\right)$; we may choose $\beta^{j}\left(x_{i}\right)$ to coincide with $\beta^{j}\left(y_{i}\right)$ since

$$
\beta^{j}{ }_{i j}\left(y_{i}\right) \geqslant\left(y_{i}+A^{i-1}{ }_{j}\right)\left(a_{i}+a_{j}\right)
$$

which implies $\beta^{j}{ }_{i j}\left(y_{i}\right) \geqslant\left(x_{i}+A^{i-1}{ }_{j}\right)\left(a_{i}+a_{j}\right)$. Now $e\left(x_{i}\right) u\left(y_{i}\right)=u\left(x_{i}\right)$ for each $i=1, \ldots, n$ and hence $M\left(x_{1}, \ldots, x_{n}\right) \leqslant M\left(y_{1}, \ldots, y_{n}\right)$. Because of (6.5) it follows that $M(x) \leqslant M(y)$.

13. Proof of (6.7). Since $x$ is a union of $i$-elements, $i=1, \ldots, n$, it is sufficient to prove (6.7) with the restriction that $x$ is an $i$-element. Then if $y_{1}, \ldots, y_{n}$ is a base-decomposition of $y, u(x)$ will have a representation

$$
u(x)=\gamma_{1} u\left(y_{1}\right)+\ldots+\gamma_{n} u\left(y_{n}\right) .
$$

Let $e(x)$ be the $i$ th coordinate of $u(x)$ and $e\left(y_{m}\right)$ the $m$ th coordinate of $u\left(y_{m}\right)$. Then $e(x) u(x)=u(x)$ and replacing $\gamma_{m}$ by $e(x) \gamma_{m}$ we may suppose $e(x) \gamma_{m}=\gamma_{m}$ for all $m$. 
Now if $x$ is an $i$-element ( $i$ fixed) and $i<n$ then the $n$th coordinates of $u(x)$ and of $\gamma_{m} u\left(y_{m}\right)$, for $m<n$, are all 0 and hence the $n$th coordinate $\gamma_{n} e\left(y_{n}\right)=0$. This implies $\gamma_{n} u\left(y_{n}\right)$ is the zero vector. Successive applications of this argument show that $\gamma_{m} u\left(y_{m}\right)=0$ for all $m>i$ and that $e(x)=\gamma_{i} e\left(y_{i}\right)$.

The last paragraph of $\S 4$ shows that $\gamma_{i} u\left(y_{i}\right)=u\left(\bar{y}_{i}\right)$ for some $i$-element $\bar{y}_{i}$. Then (4.12) shows that $\bar{y}_{i} \leqslant y_{i}$ since $\left(e\left(y_{i}\right)\right)^{r}{ }_{i} \geqslant\left(\gamma_{i} e\left(y_{i}\right)\right)^{r}{ }_{i}$. Hence we need only prove (6.7) with $y_{i}$ replaced by $\bar{y}_{i}$; thus we may suppose that $\bar{y}_{i}=y_{i}$, that is, we may suppose that $\gamma_{i}=1$, and $e\left(y_{i}\right)=e(x)=e$ (say) and $e \gamma_{m}=\gamma_{m}$ for all $m<i$. Now (6.3) shows that $u\left(y_{i}\right)+\gamma_{i-1} u\left(y_{i-1}\right)=u(z)$ for some $i$-element $z$ with $z \leqslant y_{i}+y_{i-1}$. Similarly $u(z)+\gamma_{i-2} u\left(y_{i-2}\right)=u(\bar{z})$ for some $i$-element $\bar{z}$ with $\bar{z} \leqslant z+y_{i-2} \leqslant y_{i}+y_{i-1}+y_{i-2}$. Repetition of this argument finally yields $x \leqslant y_{i}+y_{i-1}+\ldots+y_{1} \leqslant y$ as required.

This completes the proof of all statements (6.1) to (6.7) and establishes the coordinatization theorem.

Added in proof. A book, in Japanese, by F. Maeda, entitled Continuous Geometry (Tokyo, 1952), is reported to contain a simplification of von Neumann's coordinatization theorem, based on methods of K. Kodaira and S. Huruya (see Mathematical Reviews, 15 (1954), p. 540). We regret that we have not been able to see any further details of this work.

\section{REFERENCES}

1. K. D. Fryer and Israel Halperin, Coordinates in Geometry, Trans. Royal Soc. of Canada, Third Series, Section III, 48 (1954), 11-26.

2. D. Hilbert, Grundlagen der Geometrie, seventh edition (Berlin, 1930).

3. O. Veblen and J. W. Young, Projective Geometry, Vol. I (New York, 1938).

4. J. von Neumann, Continuous Geometry, Vol. I, planographed lecture notes, Institute for Advanced Study (Princeton, 1936).

5. - Continuous Geometry, Vol. II, planographed lecture notes, Institute for Advanced Study (Princeton, 1936). 